\title{
On the relationship between mass and diameter distributions in tree communities
}

\author{
James C. Stegen ${ }^{1}$ and Ethan P. White ${ }^{2 *}$ \\ ${ }^{1}$ Department of Ecology and Evolutionary Biology, University of Arizona, Tucson, AZ, 85721 \\ ${ }^{2}$ Department of Biology and the Ecology Center, Utah State University, Logan, UT, 84322
}

Running Title: Relating mass and diameter distributions

Keywords: individual size distribution, variable transformation, energetic equivalence, metabolic theory, light competition, resource partitioning

Article Type: Letter

Abstract word count: 124

Manuscript word count: 2858

Number of references: 52

Figures: 2

Supplemental Appendices: 1

*Correspondence: Ethan P. White, Department of Biology, Utah State University, 5305 Old

Main Hill, Logan, UT, 84322. Phone: 435-797-2097. Fax: 435-797-1575. E-mail:

epwhite@biology.usu.edu. 


\begin{abstract}
It has been suggested that frequency distributions of individual tree masses in natural stands are characterized by power-law distributions with exponents near $-3 / 4$, and that therefore tree communities exhibit energetic equivalence among size classes. Because the mass of trees is not measured directly, but estimated from diameter, this supposition is based on the fact that the observed distribution of tree diameters is approximately characterized by a power-law with an exponent $\approx-2$. Here we show that diameter distributions of this form are not equivalent to mass distributions with exponents of -3/4, but actually to mass distributions with exponents of -11/8. We discuss the implications of this result for the metabolic theory of ecology and for understanding energetic equivalence and the processes structuring tree communities.
\end{abstract}

Keywords: individual size distribution, size spectrum, variable transformation, energetic equivalence, metabolic theory, light competition, resource partitioning 


\section{INTRODUCTION}

The idea of energetic equivalence was first suggested by Damuth (1981). Damuth noted that because the average population density of a species, $N$, was related its mass, $M$, raised to the $-3 / 4$ power and the metabolic rate of an individual, $B$, was related to its mass raised to the $+3 / 4$ power the population energy use of a species was invariant with respect to mass (i.e.

$\left.Q=B N \mu M^{3 / 4} M^{-3 / 4} \mu M^{0}\right)$. This pattern has been dubbed the Energetic Equivalence Rule and is commonly observed at broad spatial scales for mammals, invertebrates, fish, and trees (Damuth 1987; Cyr et al. 1997; Enquist et al. 1998; Ernest et al. 2003; White et al. 2007).

It has recently been suggested that a related pattern occurs for individual-size distributions (ISDs) within forest communities. By assuming that the scaling of the distribution of plant masses within communities behaves in the same manner as the scaling of plant mass and density across communities, Enquist and Niklas (2001) suggested that the number of individuals in a mass class, $n$, should be proportional to the mass of that class raised to the $-3 / 4$ power. If this assumption is valid, then energetic equivalence occurs among mass classes within tree communities.

Because diameter, $D$, not mass, is typically measured in forest communities, Enquist and Niklas (2001) provided indirect support for energetic equivalence across mass classes by deriving a prediction for the form of the size distribution in terms of diameter, $n \mu M^{-3 / 4} \mu D^{-2}$. In support of this model, Enquist and Niklas (2001) showed that for many of Alwyn Gentry's forest plots (Phillips \& Miller 2002) abundance among size classes declines approximately to the -2 power of diameter (but see White et al. 2008). While the precise form of the empirical diameter distribution and the value of the exponent have recently been questioned, it does appear that diameter distributions of many forests can be approximately characterized by this pattern 
over some range of body sizes (Coomes et al. 2003; Muller-Landau et al. 2006b; White et al. 2008; see also Fig. 2).

This result suggests a roughly equal partitioning of resources, energy flux, and net primary production among mass and diameter classes within forests and may provide insights into the processes structuring forest communities (Enquist \& Niklas 2001; Enquist 2002). This pattern has subsequently been used to provide linkages from individual-level energetic constraints to community structure to whole ecosystem processes, and in turn, advance the metabolic theory of ecology as a powerful and predictive tool capable of integrating across levels of biological organization (Enquist et al. 2003; Brown et al. 2004; Kerkhoff \& Enquist 2006; Kerkhoff \& Enquist 2007). However, here we show that the derived form of the diameter distribution is incorrect and discuss the implications of this result for metabolic theory, energetic equivalence, and the processes structuring tree communities.

\section{RELATING MASS AND DIAMETER DISTRIBUTIONS}

Enquist and Niklas (2001) established the predicted form of the diameter distribution by first assuming that $N$ and $n$ scale with body size in the same manner (i.e. $n \mu M^{-3 / 4}$ ) and then substituting the relationship between individual mass and tree diameter, $M \mu D^{8 / 3}$ (Niklas 1994; West et al. 1997; West et al. 1999), into the predicted form of the mass distribution. This yields $n \mu M^{-3 / 4} \mu\left(D^{8 / 3}\right)^{-3 / 4} \mu D^{-2}$ (see also Coomes et al. 2003; West \& Brown 2004; Marquet et al. 2005; West \& Brown 2005; Muller-Landau et al. 2006b; Woodhouse 2006). However, this substitution is not a valid approach for transforming between variables when dealing with size distributions, which, unlike other allometric relationships, are characterized by probability 
density functions. The problem occurs because changing from mass to diameter not only changes the position of individual points, but also changes the position of those points relative to one another and hence the density of those points along the size axis.

The appropriate univariate transformation between two probability density functions $f_{X}(x)$ and $f_{Y}(y)$ where $x$ is related to $y$ by $x=h(y)$ is

$$
f_{Y}(y)=h^{\prime}(y) f_{X}(h(y))
$$

where $h^{\prime}(y)$ is the derivative of $h(y)$ with respect to $y$ (e.g., Freund 1971; Mood et al. 1974; Ross 2006). This is the standard method from calculus for a change of variables in a definite integral (Thomas \& Finney 1996). The derivative accounts for the change in the position of the points relative to one another. Therefore the predicted diameter distribution for a model where the mass distribution is characterized by $f_{M}(M) \mu M^{-3 / 4}$, and where $M$ and $D$ are related by $M \mu D^{8 / 3}$, is given by

$$
f_{D}(D) \mu D^{5 / 3} f_{M}\left(D^{8 / 3}\right) \mu D^{5 / 3}\left(D^{8 / 3}\right)^{-3 / 4} \mu D^{-1 / 3}
$$

demonstrating that the predicted relationship between abundance and diameter is actually $D^{-1 / 3}$, not $D^{-2}$. Equivalently we can determine the form of the mass distribution that corresponds to a diameter distribution with an exponent of -2 :

$$
f_{M}(M) \mu M^{-5 / 8} f_{D}\left(M^{3 / 8}\right) \mu M^{-5 / 8}\left(M^{3 / 8}\right)^{-2} \mu M^{-11 / 8}
$$

suggesting that observed individual size distributions of diameter actually support distributions of mass with exponents near $-11 / 8$, not $-3 / 4$. These results were confirmed by both simulations (Fig. 1) and analyses of empirical data (Fig. 2).

This variable transformation error has also occurred in other areas of ecology. Notably, May (1986), Peters (1983), and Southwood et al. (2006) did not properly transform between size 
variables when evaluating the form of species body size distributions. The error is likely a result of thinking about frequency distributions from the perspective of the binning based approaches often used to analyze the data. These approaches typically treat frequency distribution data as bi-variate functional relationships, making it appear that the substitution discussed above is valid. Avoiding binning based approaches when modeling and analyzing frequency distribution data may help alleviate confusion regarding the type of size-abundance relationship being studied, and thus clarify the appropriate approach to transforming between variables.

We have focused on the transformation originally laid out in Enquist and Niklas (2001). However, the form of both the individual size distribution and the relationship between diameter and mass are subjects of debate (Niklas 1994; West et al. 1997; West et al. 1999; Enquist \& Niklas 2001; Coomes et al. 2003; Chave et al. 2005; Muller-Landau et al. 2006b; Price et al. 2007). The general approach we take is valid for any proposed form of the individual size distribution and functional relationship between diameter and mass. The transformation can generally be expressed as,

$$
f_{M}(M)=\int P(M \mid D) f_{D}(D) d D .
$$

In concept this general solution makes it possible to incorporate alternative and/or more complex forms of the component relationships including the incorporation of: 1) error around the allometric relationships; 2) species level variation in the normalization (e.g., through variation in wood density; Chave et al. 2005) and exponent of the allometric function relating $M$ to $D$ (Price et al. 2007); and 3) the use of different distributions and functional relationships (e.g., those in Chave et al. 2005 and Muller-Landau et al. 2006b). In practice, analytical solutions to the transformation described in equation (2) may prove more difficult in the presence of these additional sources of complexity. Preliminary simulation results suggest that variability in the 
exponent does not affect the transformation (Appendix S1), but that error around the allometric relationships may have some influence on the results (Appendix S1). Further research will be necessary to fully understand the implications of these and other complexities for transforming between different forms of the size distribution.

\section{IMPLICATIONS FOR THE METABOLIC THEORY OF ECOLOGY}

Because the observed form of the diameter distribution in tree communities is not consistent with an individual size distribution of the form $f_{M}(M) \mu M^{-3 / 4}$, the published metabolic theory explanation for the form of the individual size distribution (Enquist \& Niklas 2001) is not supported by empirical data. Therefore metabolic theory has yet to provide a mechanistic explanation for ISDs within forests (see also Torres et al. 2001; Coomes 2006; Muller-Landau et al. 2006b). In addition, the two studies in animal communities that have offered support for the predicted -3/4 form of the individual mass distribution (Ackerman et al. 2004; Meehan 2006) did so using incorrect statistical methods (see White et al. 2008 for a detailed explanation). Specifically, a value of 1 must be subtracted from the exponent when using logarithmically binned size classes (see Bonnet et al. 2001; Andersen \& Beyer 2006; Sims et al. 2007; White et al. 2008). As a result these studies support an exponent near -7/4, not -3/4.

It has been suggested that these studies should be interpreted as deriving $f_{\log (M)}(\log (M))$, and thus actually predict $f_{M}(M) \propto M^{-7 / 4}$ (Reuman et al. in press). Regardless, we are aware of no data that support an individual size distribution with the $f_{M}(M) \mu M^{-3 / 4}$ form.

It should be noted, however, that Enquist and Niklas (2001) do provide a simulation model from which a size distribution characterized by $f_{D}(D) \mu D^{-2}$ emerges. Their model 
follows the succession of spatially explicit forest communities from an initial "seeding” of propagules to an equilibrium forest structure. Metabolic theory provides the basis for growth such that partitioning of new biomass into stems, leaves, and reproductive biomass is assumed to scale with mass as predicted by metabolic theory (West et al. 1999; Enquist et al. 2000; Enquist \& Niklas 2002; Niklas et al. 2003). In addition to purely metabolic constraints, mortality and growth in this model are also affected by light competition due to light attenuation caused by overhead canopies (see also Coomes 2006; Muller-Landau et al. 2006a; Coomes \& Allen 2007).

The simulated ISDs are well characterized by $f_{D}(D) \mu D^{-2}$ (Enquist \& Niklas 2001). Similar results are also seen in more complex simulation models with allometric foundations (Chave 1999). The rough concordance between observed and simulated distributions suggests that the rules governing biomass partitioning, as predicted by metabolic theory, may have important influences on the form of the size distribution.

In addition to predicting the form of the individual size distribution, a small branch of metabolic theory has utilized the presumed form of the mass distribution to make predictions for the scaling of ecosystem properties such as carbon storage and flux, and whole community nutrient stocks (Kerkhoff \& Enquist 2006). Determining the specific implications of our central result for this study will require re-derivation of the model and re-analysis of the affected predictions. While detailed redevelopment is beyond the scope of the current study, it is clear that within-size-class predictions for any community or ecosystem attribute will be sensitive to the assumed ISD. In addition, any model for ecosystem processes where the size distribution plays a meaningful role will be influenced by the use of the incorrect form of the ISD (e.g., Kerkhoff \& Enquist 2006). The form of the ISD is thus important for understanding how organismal processes ‘scale-up’ to populations, communities, and ecosystems. In general, our 
results impact the metabolic theory predictions for the form of the ISD and for community and ecosystem level properties based on the ISD, but our results have no implications for the network model itself (West et al. 1997; 1999) or for any predictions of individual- and species-level properties (e.g. metabolic rate, mortality rate, etc.).

\section{IMPLICATIONS FOR ENERGETIC EQUIVALENCE}

Different processes are responsible for generating the individual size distribution and the species-level size-density relationship (SDR; sensu White et al. 2007), the pattern Damuth (1981) originally proposed to exhibit energetic equivalence (Jennings et al. 2007; White et al. 2007). These two patterns also take different mathematical forms. The ISD is a frequency distribution and is thus characterized by a probability density function, while the SDR is a bi-variate functional relationship. Our results have no implications for the traditional SDR based energetic equivalence rule because if population-level metabolic rate, $Q$, is invariant with respect to body size for one size measure, by definition it is invariant with respect to the other size measures (since direct substitution is the appropriate way to change variables for the SDR). For example, given the size dependence of metabolic rate ( $B \mu M^{3 / 4} \mu D^{2}$ ), population size must decline with body size such that $Q \mu B N \mu M^{3 / 4} M^{-3 / 4} \mu M^{0}$ and $Q \mu B N \mu D^{2} D^{-2} \mu D^{0}$ (Enquist et al. 1998).

In contrast, for the individual size distribution only a single measure of size (or a set of size measures that are related isometrically) can exhibit energetic equivalence. For example, observed diameter distributions in tree communities are fairly close in many cases to exhibiting energetic equivalence while mass distributions exhibit a steep decline in energy use with increasing size class: $q \mu B n \mu D^{2} D^{-2} \mu D^{0}$ and $q \mu B n \mu M^{3 / 4} M^{-11 / 8} \mu M^{-5 / 8}$, where $q$ is 
energy flux within a size class. More generally, it is a mathematical certainty that within any community, energy will not be partitioned among size classes in the same manner for different characterizations of body size when those characterizations are related allometrically (e.g. fish mass $\sim$ fish length ${ }^{3}$ or tree mass $\sim$ tree diameter $^{8 / 3}$ ).

The size of a tree can be defined based on various measurements including mass, trunk diameter, height $(H)$, and crown area (i.e. leaf mass; $A$ ). Metabolic theory predicts, and/or empirical data support, allometric relationships between these measures of size such that $M \mu D^{8 / 3} \mu H^{4} \mu A^{4 / 3}$ (Niklas 1994; West et al. 1997; West et al. 1999; Niklas \& Enquist 2001; Muller-Landau et al. 2006a). Hence, a forest characterized by the ISDs $\mathcal{F}_{\boldsymbol{B}}(\mathbf{D}$ and

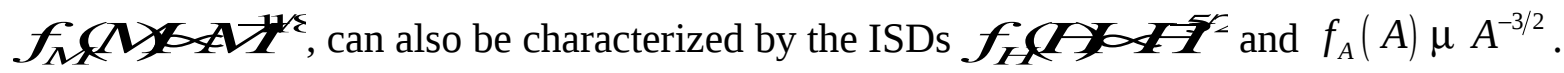
Combining these height and crown area ISDs with the dependence of metabolic rate on height ( $B \mu H^{3}$ ) and crown area ( $B \mu A$ ) shows that diameter is the only measure of size for which energetic equivalence is approximated within forests: $q \mu D^{0}, q \mu M^{-5 / 8}$, $q \mu B f_{H}(H) \mu H^{3} H^{-5 / 2} \mu H^{1 / 2}$, and $q \mu B f_{A}(A) \mu A A^{-3 / 2} \mu A^{-1 / 2}$. This occurs because the relative position of individuals along the size axis changes when transforming from one size measure to another. As a result, the number of individuals in a size class is dependent on how size is characterized, such that the amount of energy fluxed within a size class changes with the choice of size measure. This remains true regardless of taxon or community type. In contrast the amount of energy fluxed by a population or through a whole community is not dependent on how size is characterized because the number of individuals and the fluxes of each individual remain the same by definition. Thus, energetic equivalence holds across forests (Enquist et al. 1998) regardless of how size is characterized. 


\section{IMPLICATIONS FOR PROCESSES STRUCTURING TREE COMMUNITIES}

Approximately equal energy use across diameter classes may point to diameter as a functional attribute of tree morphology critical to resource partitioning among individuals. If diameter is directly related to resource partitioning, the observation of approximate energetic equivalence would represent an important clue to understanding how resources are divided within forests. However, diameter is unlikely to be the most relevant aspect of size given that canopy area, leaf mass, and tree height are more directly related to light acquisition than is trunk diameter (see also Lawes et al. 2008). In fact, the observed form of the tree size distribution has been questioned due to a presumed dominance of light acquisition by larger individuals (Coomes 2006; Muller-Landau et al. 2006b; Coomes \& Allen 2007). Dominant resource acquisition by larger individuals would suggest that tree height, the only measure of size examined here for which energy flux increases across size classes, is the aspect of size most relevant to resource partitioning in forests. However, resource acquisition should logically result from a combination of canopy area (the number of leaves) and tree height (the per leaf resource availability). While more research is needed to determine how different aspects of tree size influence resource partitioning within forests (Schwinning \& Weiner 1998), some insight is provided by the fact that realistic diameter distributions emerge from simulation models combining the ecological impacts of competition for light and stochastic mortality with allometric partitioning of biomass into growth and reproduction (Chave 1999; Enquist \& Niklas 2001). In addition, Hara (1984) hypothesized that realized ISDs are sensitive to size dependent biomass partitioning. Roughly equivalent energy flux across diameter classes may thus represent an emergent property of plant 
communities within which individuals are constrained to follow idealized biomass partitioning rules.

Exploring the sensitivity of the ISDs predicted by these models to the assumptions of individual biomass partitioning and light competition will provide a more rigorous evaluation of the processes leading to observed patterns of resource division among individuals. This will help determine if patterns of resource partitioning are due to a direct influence of size per se or if they emerge indirectly through ecological interactions and individual biomass partitioning. In addition, studying the temporal dynamics of ISDs following disturbance would allow community assembly to be examined through time from a body size perspective (Kohyama 1993). In general, understanding the relative influence of, and interactions between, individual biomass partitioning and competition should help elucidate fundamental processes structuring forested ecosystems.

\section{CONCLUSIONS}

Here we have shown that the distribution of individual tree masses predicted by Enquist and Niklas (2001), $f_{M}(M) \mu M^{-3 / 4}$, is not consistent with the observed form of tree community diameter distributions, $f_{D}(D) \mu D^{-2}$, and is thus not supported by empirical data. Therefore, when properly analyzed the current metabolic theory explanation for the form of ISDs in tree communities is readily rejected. This result also demonstrates that patterns of energy use across size classes within forests are dependent upon how size is characterized. If measured in terms of diameter, all size classes flux approximately the same amount of energy whereas energy flux declines with mass and crown area and increases with height. This implies that energy is not partitioned due to size per se, but rather due to different functional attributes of different aspects 
of size. This 'context' dependence of energy partitioning occurs because of the allometric relationships between different aspects of tree size and because individual size distributions are frequency distributions, not bi-variate functions. The nature of this dependence raises significant challenges for understanding the relative dominance of different size classes in tree communities.

\section{ACKNOWLEDGMENTS}

We are extremely grateful to Molly Stegen and Morgan Ernest for advice and support throughout the conception and development of this project. Van Savage, Jessica Green, and Tommaso Zillio kindly took the time to confirm the central result of the paper, and Hélène Morlon introduced us to the more general form of transformation given in equation (2). Several conversations with James Brown significantly improved our understanding of our results. The manuscript benefited from comments from Brian Enquist, Scott Stark, James O’Dwyer, Jessica Green, Tommaso Zillio, Kenneth Feeley, Christian Mulder, and three anonymous reviewers. The BCI forest dynamics research project was made possible by National Science Foundation grants to Stephen P. Hubbell, support from the Center for Tropical Forest Science, the Smithsonian Tropical Research Institute, the John D. and Catherine T. MacArthur Foundation, the Mellon Foundation, the Celera Foundation, and numerous private individuals, and through the hard work of over 100 people from 10 countries over the past two decades. The plot project is part the Center for Tropical Forest Science, a global network of large-scale demographic tree plots. EPW was supported by an NSF Postdoctoral Fellowship in Biological Informatics (DBI-0532847).

\section{REFERENCES}


Ackerman J.L., Bellwood D.R. \& Brown J.H. (2004). The contribution of small individuals to density-body size relationships: examination of energetic equivalence in reef fishes. Oecologia, 139, 568-571.

Andersen K.H. \& Beyer J.E. (2006). Asymptotic size determines species abundance in the marine size spectrum. Am Nat, 168, 54-61.

Bonnet E., Bour O., Odling N.E., Davy P., Main I., Cowie P., et al. (2001). Scaling of fracture systems in geological media. Reviews of Geophysics, 39, 347-383.

Brown J.H., Gillooly J.F., Allen A.P., Savage V.M. \& West G.B. (2004). Toward a metabolic theory of ecology. Ecology, 85, 1771-1789.

Chave J. (1999). Study of structural, successional and spatial patterns in tropical rain forests using TROLL, a spatially explicit forest model. Ecol Modell, 124, 233-254.

Chave J., Andalo C., Brown S., Cairns M.A, Chambers J.Q., Eamus D., et al. (2005). Tree allometry and improved estimation of carbon stocks and balance in tropical forests. Oecologia, 145, 87-99.

Condit, R. (1998). Tropical Forest Census Plots. Springer-Verlab and R. G. Landes Company, Berlin, Germany, and Georgetown, Texas.

Coomes D.A. (2006). Challenges to the generality of WBE theory. Trends Ecol Evol, 21, 593-596.

Coomes D.A. \& Allen R.B. (2007). Mortality and tree-size distributions in natural mixed-age forests. $J$ Ecol, 95, 27-40.

Coomes D.A., Duncan R.P., Allen R.B. \& Truscott J. (2003). Disturbances prevent stem size-density distributions in natural forests from following scaling relationships. Ecol Lett, 6, 980-989. 
Cyr H., Peters R.H. \& Downing J.A. (1997). Population density and community size structure: Comparison of aquatic and terrestrial systems. Oikos, 80, 139-149.

Damuth J. (1981). Population-density and body size in mammals. Nature, 290, 699-700.

Damuth J. (1987). Interspecific allometry of population-density in mammals and other animals the independence of body-mass and population energy-use. Biol J Linn Soc, 31, 193-246.

Enquist B.J. (2002). Universal scaling in tree and vascular plant allometry: Toward a general quantitative theory linking plant form and function from cells to ecosystems. Tree Physiology, 22, 1045-1064.Enquist B.J., Brown J.H. \& West G.B. (1998). Allometric scaling of plant energetics and population density. Nature, 395, 163-165.

Enquist B.J., Economo E.P., Huxman T.E., Allen A.P., Ignace D.D. \& Gillooly J.F. (2003).

Scaling metabolism from organisms to ecosystems. Nature, 423, 639-642.

Enquist B.J. \& Niklas K.J. (2001). Invariant scaling relations across tree-dominated communities. Nature, 410, 655-660.

Enquist B.J. \& Niklas K.J. (2002). Global allocation rules for patterns of biomass partitioning in seed plants. Science, 295, 1517-1520.

Enquist B.J., West G.B. \& Brown J.H. (2000). Quarter-power allometric scaling in vascular plants: Functional basis and ecological consequences. Oxford University Press, New York.

Ernest S.K.M., Enquist B.J., Brown J.H., Charnov E.L., Gillooly J.F., Savage V.M., et al. (2003). Thermodynamic and metabolic effects on the scaling of production and population energy use. Ecol Lett, 6, 990-995.

Freund J.E. (1971). Mathematical Statistics. 2nd edn. Prentice-Hall, Englewood Cliffs, New Jersey. 
Hara T. (1984). A stochastic model and the moment dynamics of the growth and size distribution in plant populations. $J$ Theor Biol, 109, 173-190.

Hubbell, S.P., Condit, R., \& Foster, R.B. (2005). Barro Colorado Forest Census Plot Data. URL http://ctfs.si/edu/datasets/bci.

Hubbell S.P., Foster R.B., O’Brien S.T., Harms K.E., Condit R., Wechsler B., Wright S.J., \& Loo de Lao S. (1999). Light gap disturbances, recruitment limitation, and tree diversity in a neotropical forest. Science, 283, 554-557.

Jennings S., De Oliveira J.A.A. \& Warr K.J. (2007). Measurement of body size and abundance in tests of macroecological and food web theory. J Anim Ecol, 76, 72-82.

Kerkhoff A.J. \& Enquist B.J. (2006). Ecosystem allometry: the scaling of nutrient stocks and primary productivity across plant communities. Ecol Lett, 9, 419-427.

Kerkhoff A.J. \& Enquist B.J. (2007). The implications of scaling approaches for understanding resilience and reorganization in ecosystems. Bioscience, 57, 489-499.

Kohyama T. (1993). Size-structured tree populations in gap-dynamic forests - The forest architecture hypothesis for the stable coexistence of species. J Ecol 81, 131-143.

Lawes M.J., Griffiths M.E., Midgley J.J., Boudreau S., Eeley H.A.C., \& Chapman C.A. (2008). Tree spacing and area of competitive influence do not scale with tree size in an African rain forest. J Veg Sci, 19, 729-738.

Marquet P.A., Quinones R.A., Abades S., Labra F., Tognelli M., Arim M., et al. (2005). Scaling and power-laws in ecological systems. J Exp Biol, 208, 1749-1769.

May R.M. (1986). The search for patterns in the balance of nature: Advances and retreats. Ecology, 67,1116-1126. 
Meehan T.D. (2006). Energy use and animal abundance in litter and soil communities. Ecology, 87, 1650-1658.

Mood A.M., Graybill F.A. \& Boes D.C. (1974). Introduction to the Theory of Statistics. 3rd edn. McGaw-Hill, New York.

Muller-Landau H.C., Condit R.S., Chave J., Thomas S.C., Bohlman S.A., Bunyavejchewin S., et al. (2006a). Testing metabolic ecology theory for allometric scaling of tree size, growth and mortality in tropical forests. Ecol Lett, 9, 575-588.

Muller-Landau H.C., Condit R.S., Harms K.E., Marks C.O., Thomas S.C., Bunyavejchewin S., et al. (2006b). Comparing tropical forest tree size distributions with the predictions of metabolic ecology and equilibrium models. Ecol Lett, 9, 589-602.

Niklas K.J. (1994). Plant allometry: The scaling of form and process. University of Chicago Press, Chicago.

Niklas K.J. \& Enquist B.J. (2001). Invariant scaling relationships for interspecific plant biomass production rates and body size. Proc Natl Acad Sci USA, 98, 2922-2927.

Niklas K.J., Midgley J.J. \& Enquist B.J. (2003). A general model for mass-growth-density relations across tree-dominated communities. Evol Ecol Res, 5, 459-468.

Peters R.H. (1983). The ecological implications of body size. Cambridge University Press, New York, NY.

Phillips O. \& Miller J.S. (2002). Global patterns of plant diversity: Alwyn Gentry's forest transect dataset. Monographs in Systematic Botany from the Missouri Botanical Garden, 89.

Price C.A., Enquist B.J. \& Savage V.M. (2007). A general model for allometric covariation in botanical form and function. Proc Natl Acad Sci USA, 104, 13204-13209. 
Ross S. (2006). A First Course in Probability. Seventh edn. Pearson Prentice Hall, Upper Saddle River, New Jersey.

Schwinning, S., \& J. Weiner. 1998. Mechanisms determining the degree of size asymmetry in competition among plants. Oecologia, 113, 447-455.

Sims D.W., Righton D. \& Pitchford J.W. (2007). Minimizing errors in identifying Levy flight behaviour of organisms. J Anim Ecol, 76, 222-229.

Southwood T.R.E., May R.M. \& Sugihara G. (2006). Observations on related ecological exponents. Proc Nat Acad Sci USA, 103, 6931-6933.

Thomas G.B.J. \& Finney R.L. (1996). Calculus. Addison-Wesley Publishing Company, Reading, Massachusetts.

Torres J.L., Sosa V.J., Equihua M. \& Torres L. (2001). On the conceptual basis of the self-thinning rule. Oikos, 95, 544-548.

West G.B. \& Brown J.H. (2004). Life's universal scaling laws. Physics Today, 57, 36-42.

West G.B. \& Brown J.H. (2005). The origin of allometric scaling laws in biology from genomes to ecosystems: towards a quantitative unifying theory of biological structure and organization. J Exp Biol, 208, 1575-1592.

West G.B., Brown J.H. \& Enquist B.J. (1997). A general model for the origin of allometric scaling laws in biology. Science, 276, 122-126.

West G.B., Brown J.H. \& Enquist B.J. (1999). A general model for the structure and allometry of plant vascular systems. Nature, 400, 664-667.

White E.P., Enquist B.J. \& Green J.L. (2008). On estimating the exponent of power-law frequency distributions. Ecology, 89, 905-912. 
White E.P., Ernest S.K.M., Kerkhoff A.J. \& Enquist B.J. (2007). Relationships between body size and abundance in ecology. Trends Ecol Evol, 22, 323-330.

Woodhouse I.H. (2006). Predicting backscatter-biomass and height-biomass trends using a macroecology model. IEEE Transactions on Geoscience and Remote Sensing, 44, 871-877. 


\section{FIGURE LEGENDS}

Figure 1. Simulation results confirming the analytical solutions for transformations between different measures of body size. Random datasets of 500 individuals each were generated from power-law distributions with the appropriate exponents, a minimum value of 1 and a maximum value of 10,000 . These datasets were treated as either mass data (exponent $=-3 / 4$ ) or diameter data (exponent $=-2)$. The exponent of each dataset was determined using both maximum likelihood estimation (solid lines) and normalized logarithmic binning (dashed lines), the values were converted to the alternate measure of size (diameter or mass) using $M \mu D^{8 / 3}$, and finally the exponent for the alternate size measure was determined. Distributions of the results of 10,000 of these simulations are presented for (a) Converting from mass (exponent $=-3 / 4$; blue lines) to diameter (red lines; analytical solution for exponent $=-1 / 3$ ) and (b) converting from diameter (exponent $=-2$; red lines) to mass (blue lines; analytical solution for exponent $=-11 / 8$ ). The simulated data clearly replicate the analytical result.

Figure 2. Empirical results confirming the analytical solutions for transformations between different measures of body size and rejecting the previously assumed distribution:

$f_{M}(M) \mu M^{-3 / 4}$. Data from the 50ha plot at Barro Colorado Island (Condit 1998; Hubbell et al. 1999; Hubbell et al. 2005) were subdivided into 50 1ha subplots. Diameters (red lines) where converted to masses (blue lines) using $M \mu D^{8 / 3}$. Power-laws were fit to the data using maximum likelihood estimation based on the Pareto distribution (solid lines) and the Truncated Pareto distribution (dotted lines; using the maximum value of diameter or mass for the entire 50ha plot as the maximum attainable value). The mode of the Pareto diameter distribution exponents is indicated by the solid black line and the predicted value for the mass exponent based on this observed diameter exponent is indicated by the dashed black line. 
Figure 1
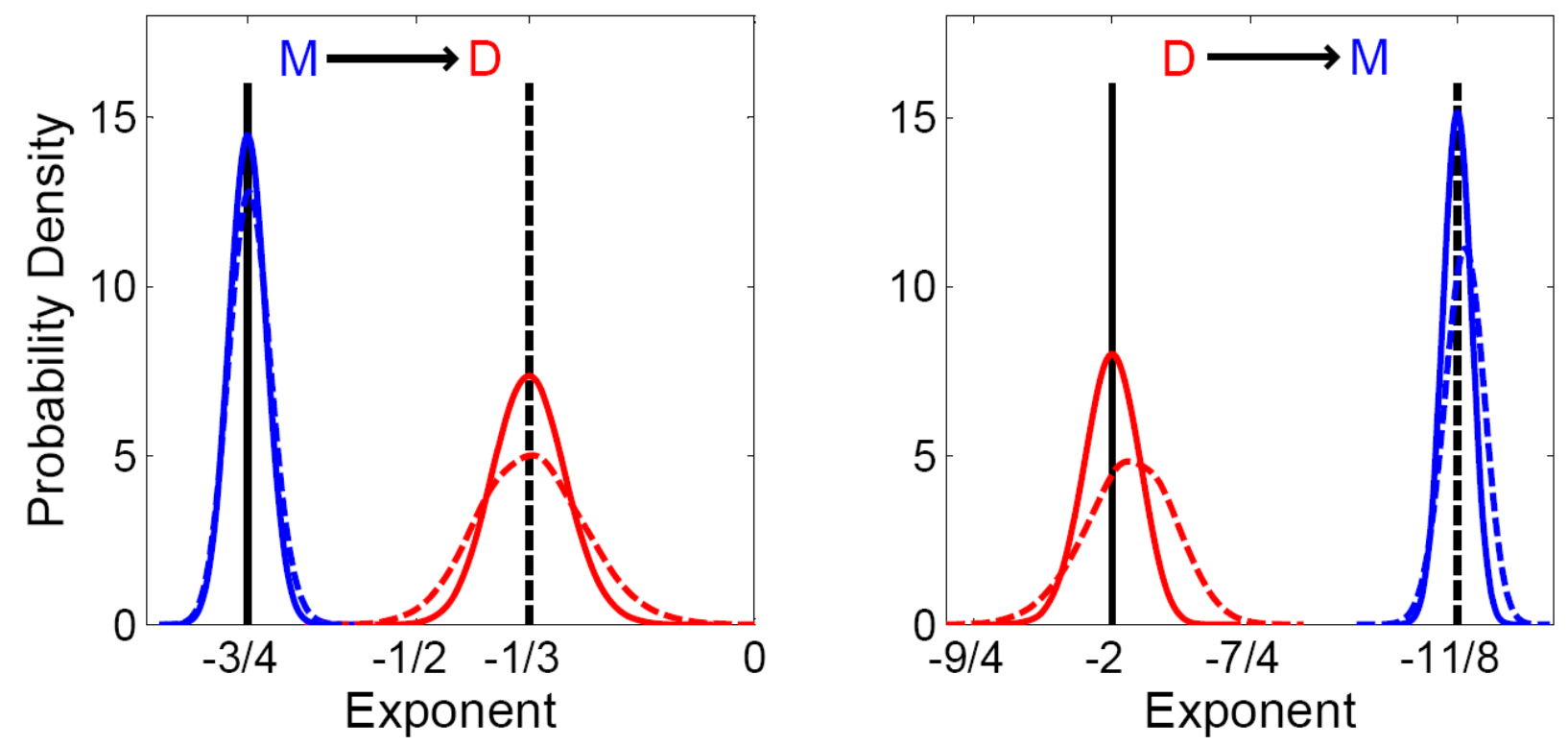
Figure 2

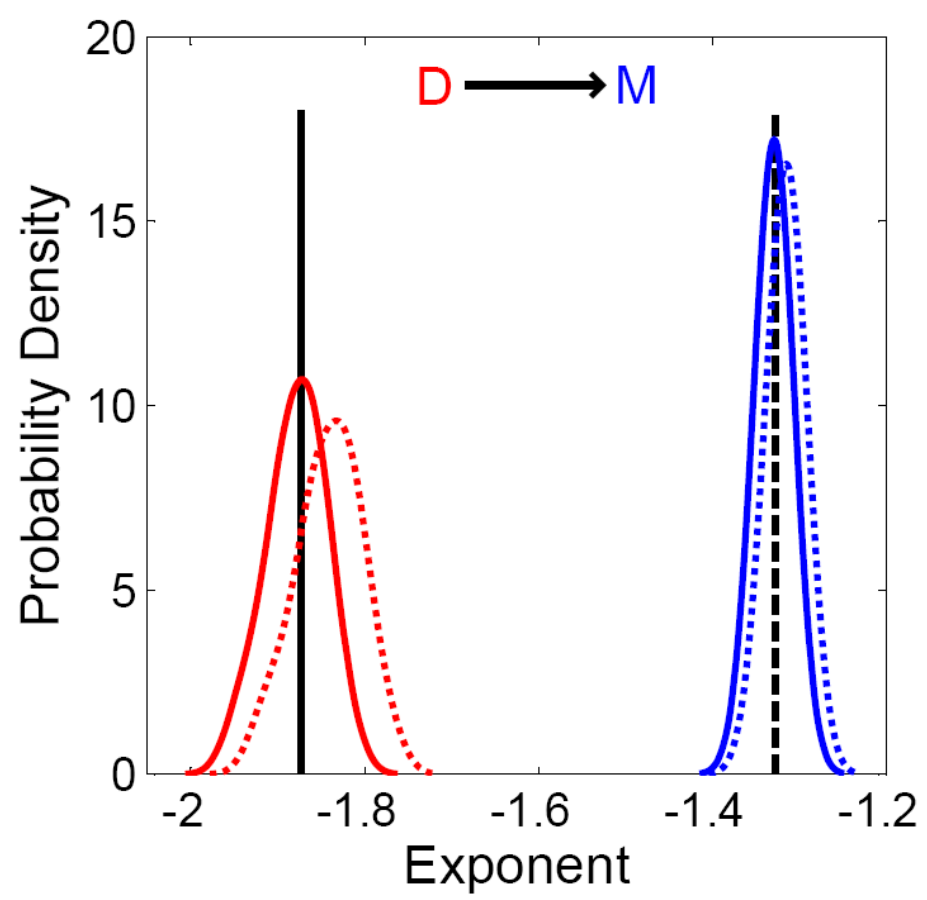

